\title{
ADDED MASS AND DAMPING OF A \\ VIBRATING ROD IN CONFINED \\ VISCOUS FLUIDS
}

by

M. W. Wambsganss, S. S. Chen, and J. A. Jendrzejczyk

Components Technology Division
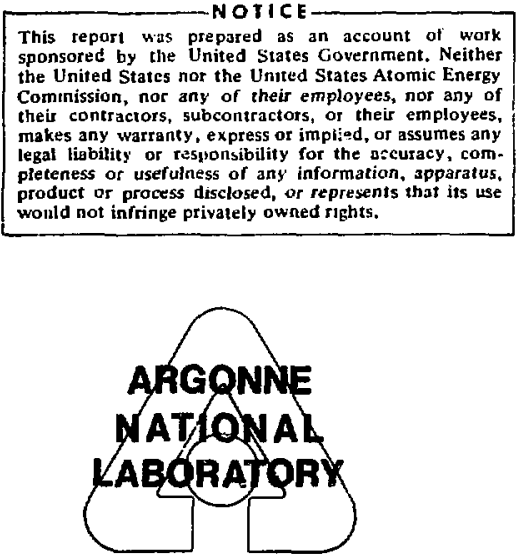

U OF C-AUA-USAEC

BASE TECHNOLOGY

- September 1974 
The facilities of Argonne National Laboratory are owned by the United States Government. Under the terms of a contract (W-31-109-Eng-38) between the U. S. Atomic Energy Coinmission, Argonne Universities Association and The University of Chicago, the University employs the staff and operates the Laboratory in accordance with policies and programs formulated, approved and reviewed by the Association.

\section{MEMBERS OF ARGONNE UNIVERSITIES ASSOCIATION}

The University of Arizona Carnegie-Mellon University Case Western Reserve University The University of Chicago University of Cincinnati Illinois Institute of Technology

University of Illinois

Indiana University

Iowa State University

The University of lowa
Kansas State University The University of Kansas Loyola University Marquette University Michigan State University The University of Michigan University of Minnesota University of Missouri Northwestern University University of Notre Dame
The Ohio State University

Ohio University

The Pennsylvania State University

Purdue University

Saint Louis University

Southern Illinois University

The University of Texas at Austin

Washington University

Wayne State University

The University of Wisconsin

\section{NOTICE}

This report was prepared as an account of work sponsored by the United States Government. Neither the United States nor the United States Atomic Energy Commission, nor any of their employees, nor any of their contractors, subcontractors, or their employees, makes any viarranty, express or implied, or assumes any legal liability or responsibility for the accuracy, completeness or usefulness of any information, apparatus, product or process disclosed, or represents that its use would not infringe privately-owned rights.

Printed in the United States of America Available from

National Technicai Information Service

U.S. Department of Commerce 5285 Port Royal Road

Springfield, Virginia 22151

Price: Printed Copy \$4.00; Microfiche $\$ 2.25$ 


\section{TABLE OF CONTENTS}

\section{$\underline{\text { Page }}$}

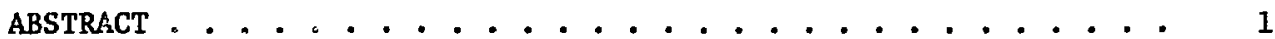

I. INTRODUCTION . . . . . . . . . . . . . . . . . 1

II. THEORY ......................... 2

A. Formulation of the Governing Equations . . . . . . . 2

B. Solution to the Equations .............. 4

C. Added Mass and Damping Factors . . . . . . . . . 10

III. EXPERIMENT ..................... 13

A. Description of Experiment . . . . . . . . . 13

B. Measurement Methods ................ 14

C. Test Procedure and Results .............. 16

IV. CONCLUDING REMARKS . . . . . . . . . . . . . . . 21

APPENDIX: Effective Mass Per Unit Length of Rod . . . . . . . . 23

REFERENCES ......................... . . . . . . . . . . . 


\section{LIST OF FIGURES}

No. Title

Page

1

2

3

4

5

6

6

Schematic and Coordinate System for Cylinder Vibrating in Fluid Annulus

3

Real Values of $\mathrm{H}$ as a Function of $\mathrm{D} / \mathrm{d}$ for Selected Values of $\mathbf{s}$

11

Imaginary Values of $\mathrm{H}$ as a Function of $\mathrm{D} / \mathrm{d}$ for Selected Values of $S$

Theoretical and Experimental Values of $C_{M}$ as a Function of $\mathrm{D} / \mathrm{d}$

Theoretical and Experimental Values of $\zeta_{\mathfrak{n}}$ as a Function of $\mathrm{D} / \mathrm{d}$

Effective-Radius Ratio for Hexagonal Array of Rods

22

\section{LIST OF TABLES}

1

Test Fluid Properties

14

2 


\section{ADDED MASS AND DAMPING OF A \\ VIBRATING ROD IN CONFINED \\ VISCOUS FLUIDS}

by

M. W. Wambsganss, S. S. Chen, and J. A. Jendrzejczyk

\section{ABSTRACT}

An analytical and experimental study of a cylindrical rod vibrating in a viscous fluid enclosed by a rigid, concentric cylindrical shell is presented. A closed form solution for the added mass and damping coefficient is obtained and a series of experiments with cantilevered rods vibrating in various viscous fluids is performed. Experimental data and the are in good agreement.

\section{INTRODUCTION}

When a structuraI component vibrates in a viscous fluid, the presence of the fluid gives rise to a fluid reaction force which can be interpreted as an added mass and a daming contribution to the dynamic response of the component. Added mass and damping are known to be dependent on fluid properties (in particular, fluid density and viscosity) as well as to be functions of component geometry and adjacent boundaries, whether rigid or elastic. Nuclear reactor components are typically immersed in a liquid coolant environment and, also, are often closely spaced. Many reactor internal components (fuel pins, instrument lines, measurement probes) and plant components (heat exchanger tubes) are long, slender, beam-like components which are susceptible to excitation by the coolant flow; potential flow excitation mechanisms include: forceexcitation due to random pressure fluctuations, fluidelastic instabilities, and resonant vibration associated with a coincidence between component natural frequencies and vortex-shedding or other flow-related characteristic frequencies. In analyzing the vibration response to these excitations, added mass and damping are important considerations. In general, the added mass will decrease the component natural frequencies; it thus can have a significant effect on the response and the potential for large amplitude motion caused by $a$ resnnance or instability. While damping is generally not important for "off-resonance" excitation by harmonic drivirg forces, it is important in predicting component response to broad-band random excitation in which energy is contained over a wide frequency range and damping controls the response amplitude [1] ${ }^{1}$. Damping is also an important parameter in characterizing the onset of fluidelastic instability as determined by a critical flow velocity at which the energy extracted from the flow equals the energy dissipated by damping [2].

Studies of added mass can be traced back to Stokes [3]. A brief survey was prfisented by Muga and Wilson [4]. At present, the added mass of a structural component with a simple geometry immersed in an invisaid fluid can

${ }^{1}$ Numbers in brackets refer to similarly numbered references at end of report. 
be calculated rather easily or can be found in literature [5]. However, the added mass of a system consisting of several structural components in confined viscous fluids, in general, is not available. To the writers' knowledge, there have been only a few studies considering the effects of viscous fluid on vibrating structural components: Ackermann and Arbhabhirama [6] determined experimentally the added mass of a sphere oscillating in a fluid filling a fixed concentric spherical she11; Miller [7] presented an experimental study on the dependence of hydrodynamic mass on frequency and amplitude of oscillation for a body undergoing a driven oscillatory motion; Fritz and Kiss [8] and Fritz [9] presented a theoretical analysis and test results which describe the response of a rod surrounded by a fluid annulus; and Keane [10], in an attempt to predict the dynamic response of a circular cantilever tube surrounded by a viscous fluid, provides a discussion of theoretical approaches to solving the Navier-Stokes equations for the two-dimensional incompressible fluid motion, as well as presents results from experiments involving cantilever tubes excited by harmonic driving forces.

In this report an appropriate form of the Navier-Stokes equation is solved to give the radial and tangential velocity components of the viscous flow in the fluid annulus formed by a vibrating rod and rigid containment she11. These velocity components are used to obtain representations for the fluid stresses which are, in turn, integrated over the circumference of the rod to determine the fluid reaction force. The resulting force consists of two parts: (1) a force in phase with the acceleration which acts as an added mass and (2) a drag force which acts to oppose cylinder motion and can be considered as a damping force. Coefficients of these forces are presented as functions of the containment-shell to rod radius ratio (D/d) and the dimensionless number $\left(S=\omega \mathrm{d}^{2} / v\right)$ which may easily be recognized as the product of the Reynolds and Strouhal numbers. Results are reported of experiments in which cantilevered rods are oscillated in liquids confined by rigid boundaries consisting of 1) concentric, circular cylindrical shells of various diameters, and 2) hexagonal arrays of six similar rods. The experimentally-determined added mass and damping data are in good agreement with theoretical predictions for the case of fluid annuli. Results from the hexagonal array tests axe correlated with the theoretical results by a correction factor which gives an effective radius ratio.

\section{THEORY}

\section{A. Formulation of the Governing Equations}

Consider an infinitely long cylinder of radius d oscillating along a diameter in a viscous fluid annulus (Fig. 1). The Navier-Stokes equations, governing the motion of the viscous fluid, can be cxpressed in a circular cylindrical coordinate $(r, \theta, z)$ system. Let the velocity of the cylinder be Ueiwt along $\theta=0$; the exterior wa.l 1 at $r=D$ is stationary. For small amplitudes the equations of state and motion can be linearized. Letting $u$ and $v$ denote the velocity components in the $r$ and $\theta$ directions, respectively, the equations of motion for the fluid are [11]: 


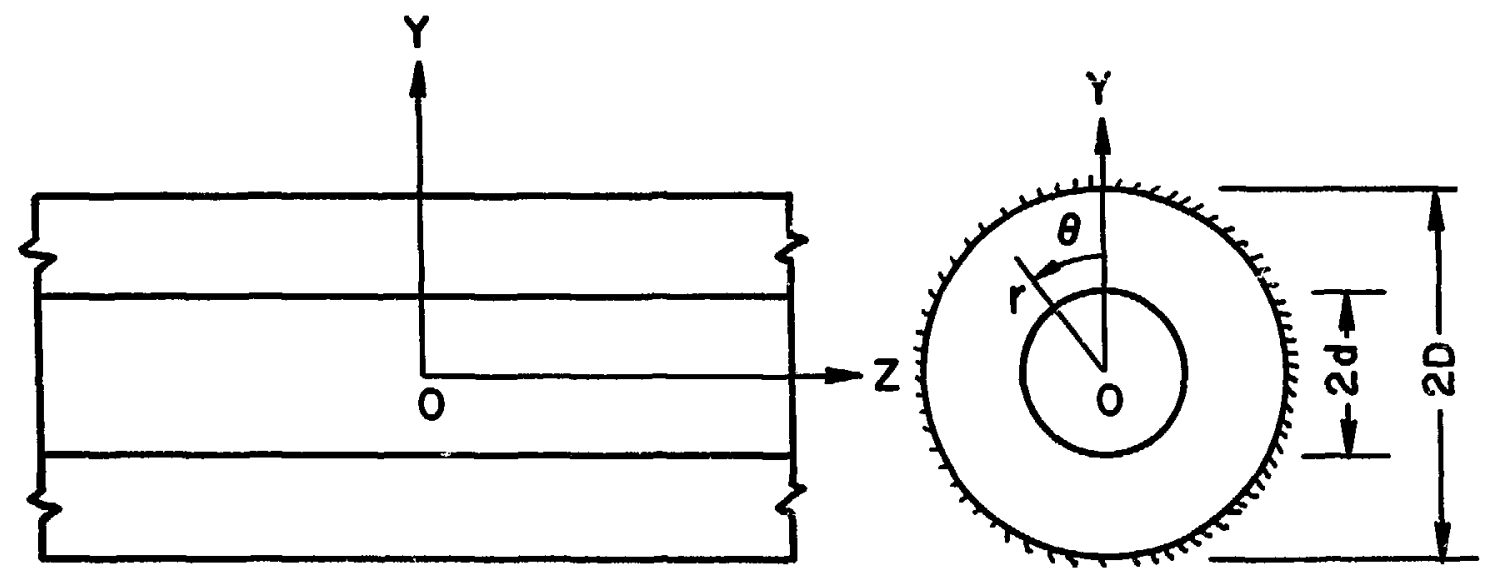

Fig. 1. Schematic and Coordinate System for Cylinder Vibrating In Flufd Annulus 


$$
\rho \frac{\partial u}{\partial t}=-\frac{\partial p}{\partial r}+\mu\left(\frac{\partial^{2} u}{\partial r^{2}}+\frac{1}{r} \frac{\partial u}{\partial r}-\frac{u}{r^{2}}+\frac{1}{r^{2}} \frac{\partial^{2} u}{\partial \theta^{2}}-\frac{2}{r^{2}} \frac{\partial v}{\partial \theta}\right),
$$

and

$$
\rho \frac{\partial v}{\partial t}=-\frac{1}{r} \frac{\partial p}{\partial \theta}+\mu\left(\frac{\partial^{2} v}{\partial r^{2}}+\frac{1}{r} \frac{\partial v}{\partial r}-\frac{v}{r^{2}}+\frac{1}{r^{2}} \frac{\partial^{2} v}{\partial \theta^{2}}+\frac{2}{r^{2}} \frac{\partial u}{\partial \theta}\right),
$$

where $\rho$ is fluid density, $\mu$ is absolute fluid viscosity, and $p$ is fluid pressure. The fluid stress components are:

$$
\begin{aligned}
& \tau_{r r}=-p+2 \mu \frac{\partial u}{\partial r}, \\
& \tau_{\theta \theta}=-p+2 \mu\left(\frac{1}{r} \frac{\partial v}{\partial \theta}+\frac{u}{r}\right),
\end{aligned}
$$

and

$$
\tau_{r \theta}=\mu\left[r \frac{\partial}{\partial r}\left(\frac{v}{r}\right)+\frac{1}{r} \frac{\partial u}{\partial \theta}\right] .
$$

Assuming an incompressible fluid, the continuity equation becomes

$$
\frac{\partial u}{\partial \mathbf{r}}+\frac{\mathrm{u}}{\mathbf{r}}+\frac{1}{\mathrm{r}} \frac{\partial \mathrm{v}}{\partial \theta}=0
$$

The velocity of the fluid at the cylinder surface must be in the direction of oscillation, so that the conditions to be satisfied by $u$ and $v$ on the cylinder are

$$
u=u \cos \theta \mathrm{e}^{i \omega t},
$$

and

$$
v=-U \sin \theta e^{i \omega t},
$$

at $r=d$. At $r=D$, the fluid velocity is zero, thus

$$
\mathbf{u}=\mathbf{v}=\mathbf{0}
$$

Equations (1) to (5) are the complete mathematical statement of the problem.

\section{B. Solution to the Equations} such that

From equation ( 3 ) it is seen that a scalar function $\psi(r, \theta, t)$ exists

$$
\mathrm{u}=-\frac{\partial \psi}{\mathrm{r} \partial \theta} \quad ; \quad \mathrm{v}=\frac{\partial \psi}{\partial \mathbf{r}}
$$




\section{$-5-$}

On substituting equations (6) into equations (1), and then eliminating p from the resulting equations, we obtain

$$
\nabla^{4} \psi-\frac{1}{v} \frac{\partial}{\partial t} \nabla^{2} \psi=0
$$

where

$$
\nu=\mu / \rho,
$$

and

$$
\nabla^{2}=\frac{\partial^{2}}{\partial r^{2}}+\frac{1}{r} \frac{\partial}{\partial r}+\frac{1}{r^{2}} \frac{\partial^{2}}{\partial \theta^{2}}
$$

The general solution of $\psi$ is

$$
\psi=\psi_{1}+\psi_{2}
$$

where $\psi_{1}$ and $\psi_{2}$ satisfy the following equations

$$
\nabla^{2} \psi_{1}=0
$$

and

$$
\nabla^{2} \psi_{2}-\frac{1}{\nu} \frac{\partial \psi_{2}}{\partial t}=0
$$

Th form of the boundary conditions, equations (4) and (5), suggests that

$$
\psi_{1}=F_{1}(r) \sin \theta e^{i \omega t},
$$

and

$$
\psi_{2}=F_{2}(r) \sin \theta e^{i \omega t}
$$

Substitution of equations (11) into equations (10) yields

$$
\frac{d^{2} F_{1}}{d r^{2}}+\frac{1}{r} \frac{d F_{1}}{d r}-\frac{1}{r^{2}} F_{1}=0,
$$

and

$$
\frac{d^{2} F_{2}}{d r^{2}}+\frac{1}{r} \frac{d F_{2}}{d r}-\frac{1}{r^{2}}+k^{2}, F_{2}=0
$$


where

$$
k=\sqrt{1 \frac{\omega}{v}}
$$

The solutions of equations (12) are

$$
F_{1}=U\left(\frac{A^{2}}{r}+B r\right) \text {, }
$$

and

$$
\mathrm{F}_{2}=\operatorname{Ud}\left[\mathrm{CI}_{1}(\mathrm{kr})+\mathrm{DK}_{1}(\mathrm{kr})\right] \text {, }
$$

where $A, B, C$, and $D$ are arbitrary constants and $I$ and $K$ are modified Bessel functions. It follows that

$$
u=v\left[-A\left(\frac{d}{r}\right)^{2}-B-C\left(\frac{d}{r}\right) I_{1}(k r)-D\left(\frac{d}{r}\right) K_{1}(k r)\right] \cos \theta e^{i \omega t},
$$

and

$$
\begin{aligned}
v=U\left\{-A\left(\frac{d}{r}\right)^{2}\right. & +B+C\left[(k d) I_{0}(k r)-\left(\frac{d}{r}\right) I_{1}(k r)\right] \\
& \left.+D\left[-(k d) k_{0}(k r)-\left(\frac{d}{r}\right) k_{1}(k r)\right]\right\} \sin \theta e^{i \omega t} .
\end{aligned}
$$

The constants $A, B, C$, and $D$ are determined from the boundary conditions. Substitution of equations (15) into equations (4) and (5) gives

$$
\begin{aligned}
& A+B+I_{1}(\alpha) C+K_{1}(\alpha) D=-1, \\
& A-B-\left[\alpha I_{0}(\alpha)-I_{1}(\alpha)\right] C+\left[\alpha K_{0}(\alpha)+K_{1}(\alpha)\right] D=1, \\
& \gamma^{2} A+B+\gamma I_{1}(\beta) C+\gamma K_{1}(\beta) D=0,
\end{aligned}
$$

and

$$
\gamma^{2} A-B-\left[\alpha I_{0}(\beta)-\gamma I_{1}(\beta)\right] C+\left[\alpha K_{0}(\beta)+\gamma K_{1}(\beta)\right] D=0,
$$

where

$$
\begin{aligned}
& \alpha=\mathbf{k d}, \\
& \beta=\mathbf{k d},
\end{aligned}
$$

and

$$
\gamma=\mathbf{d} / \mathbf{D}
$$


Solving equations (16) gives

$$
\begin{aligned}
A= & -\alpha{ }^{2}\left[I_{0}(\alpha) K_{0}(\beta)-I_{0}(\beta) K_{0}(\alpha)\right]+2 \alpha\left[I_{1}(\alpha) K_{0}(\beta)+I_{0}(\beta) K_{1}(\alpha)\right] \\
& \left.-2 \alpha \gamma\left[I_{0}(\alpha) K_{1}(\beta)+I_{1}(\beta) K_{0}(\alpha)\right]+4 \gamma\left[I_{1}(\alpha) K_{1}(\beta)-I_{1}(\beta) K_{1}(\alpha)\right]\right\} / \Delta, \\
B= & \left\{2 \alpha \gamma\left[I_{1}(\beta) K_{0}(\beta)-I_{0}(\beta) K_{1}(\beta)\right]+\alpha^{2} \gamma^{2}\left[I_{0}(\alpha) K_{0}(\beta)-I_{0}(\beta) K_{0}(\alpha)\right]\right. \\
& \left.-2 \alpha \gamma^{2}\left[I_{1}(\alpha) K_{0}(\beta)+I_{0}(\beta) K_{1}(\alpha)\right]\right\} / \Delta, \\
C= & \left\{-2 \alpha K_{0}(\beta)-4 \gamma K_{1}(\beta)+\gamma^{2}\left[2 \alpha K_{0}(\alpha)+4 K_{1}(\alpha)\right]\right\} / \Delta, \\
D= & \left\{-2 \alpha I_{0}(\beta)+4 \gamma I_{1}(\beta)+\gamma^{2}\left[2 \alpha I_{0}(\alpha)-4 I_{1}(\alpha)\right]\right\} / \Delta,
\end{aligned}
$$

and

$$
\begin{aligned}
\Delta= & \alpha^{2}\left(1-\gamma^{2}\right)\left[I_{0}(\alpha) \mathrm{K}_{0}(\beta)-\mathrm{I}_{0}(\beta) \mathrm{K}_{0}(\alpha)\right] \\
& +2 \alpha \gamma\left[\mathrm{I}_{0}(\alpha) \mathrm{K}_{1}(\beta)-\mathrm{I}_{1}(\beta) \mathrm{K}_{0}(\beta)+\mathrm{I}_{1}(\beta) \mathrm{K}_{0}(\alpha)-\mathrm{I}_{0}(\beta) \mathrm{K}_{1}(\beta)\right] \\
& +2 \alpha \operatorname{\alpha r}^{2}\left[\mathrm{I}_{0}(\beta) \mathrm{K}_{1}(\alpha)-\mathrm{I}_{0}(\alpha) \mathrm{K}_{1}(\alpha)+\mathrm{I}_{1}(\alpha) \mathrm{K}_{0}(\beta)-\mathrm{I}_{1}(\alpha) \mathrm{K}_{0}(\alpha)\right]
\end{aligned}
$$

The fluid pressure can be obtained from the second of equations (1). On substituting equations (15) into equations (1) and integrating with respect to $\theta$, we obtain

$$
\begin{aligned}
p=p_{0}-\frac{\mu U}{d} & \left\{\left[-2 \alpha\left(\frac{d}{r}\right)+(k r) \alpha^{2}+2\left(\frac{d}{r}\right)^{2}(k r)\right] I_{0}(k r)-\alpha^{2} I_{1}(k r)\right) C \\
& \left.+\left(\left[2 \alpha\left(\frac{d}{r}\right)-(k r) \alpha^{2}-2\left(\frac{d}{r}\right)^{2}(k r)\right] K_{0}(k r)-\alpha^{2} K_{1}(k r)\right) D\right\} \cos \theta e^{i \omega t} \\
& -i p \omega U d\left\{\left(\frac{d}{r}\right) A-\left(\frac{r}{d}\right) B-\left[\left(\frac{r}{d}\right) \alpha I_{0}(k r)-I_{1}(k r)\right] C\right. \\
& \left.+\left[\left(\frac{r}{d}\right) \alpha K_{0}(k r)+K_{1}(k r)\right] D\right\} \cos \theta e^{i \omega t}
\end{aligned}
$$

here, $p_{0}$ is the undisturbed fluid pressure. Finally, substitution of equations (15) Into equations (2) yields

$$
\begin{aligned}
\tau_{r r}=-p+2 \mu\left(\frac{U}{d}\right) & \left\{2\left(\frac{d}{r}\right)^{3} A+\left[-\alpha\left(\frac{d}{r}\right) I_{0}(k r)+2\left(\frac{d}{r}\right)^{2} I_{1}(k r)\right] C\right. \\
& {\left.\left[\alpha\left(\frac{d}{r}\right) K_{0}(k r)+2\left(\frac{d}{r}\right)^{2} k_{1}(k r)\right] D\right\} \cos \theta e^{I \omega t}, }
\end{aligned}
$$




$$
\begin{aligned}
\tau_{\theta \theta}=-p+2 \mu\left(\frac{U}{d}\right)\{ & -2\left(\frac{d}{r}\right)^{3} A+\left[\alpha\left(\frac{d}{r}\right) I_{0}(k r)-2\left(\frac{d}{r}\right)^{2} I_{1}(k x)\right] c \\
+ & {\left.\left[-\alpha\left(\frac{d}{x}\right) k_{0}(k r)-2\left(\frac{d}{r}\right)^{2} k_{1}(k r)\right] D\right\} \cos \theta e^{ \pm \omega t}, }
\end{aligned}
$$

and

$$
\begin{aligned}
\tau_{r \theta}=\mu\left(\frac{J}{d}\right) & \left\{4\left(\frac{d}{r}\right)^{3} A+-2 \alpha\left(\frac{d}{r}\right) I_{0}(k r)+\left(\alpha^{2}+\frac{4 d^{2}}{r^{2}}\right) I_{1}(k r)\right] c \\
& \left.+\left[2 \alpha\left(\frac{d}{r}\right) k_{0}(k r)+\left(\alpha^{2}+\frac{4 d^{2}}{r^{2}}\right) k_{1}(k r)\right] D\right\} \sin \theta e^{i \omega t} .
\end{aligned}
$$

We are now In a position to calculate the added mass and drag force. The resultant force per unit length of a cylinder is

$$
F=d \int_{0}^{2 \pi}\left(\left.\tau_{r r}\right|_{r=d} \cos \theta-\left.\tau_{r} \theta\right|_{r=d} \sin \theta\right) d \theta
$$

On substituting equations (20) into equation (21) and carrying out the integration, we obtain

$$
F=-i M \omega H e^{i \omega t},
$$

where

$$
M=\rho \pi d^{2},
$$

and

$$
H=-A+B+C I_{1}(\alpha)+D K_{1}(\alpha)
$$

$M$ is the mass of fluid per unit length displaced by the cylinder. From the first of equations (16), H may be simplified to obtain

$$
\mathrm{H}=-1-2 \mathrm{~A} \text {. }
$$

The real part of equation (22) gives

$$
F=\operatorname{MU} \omega[\operatorname{Re}(H) \sin \omega t+\operatorname{Im}(H) \cos \omega t] .
$$

In the absence of fluid, the force per unit length required to move a cylinder of mass $m$ is -mwJ sinwt. Equation (25) shows that, in addition, tyo forces are required: (1) $-M U \omega R e(H)$ sinwt, in phase with the acceleration, arises because the fluid is necessarily moved as the cylinder vibrates; the quantity $\mathrm{MRe}(\mathrm{H})$ is called the added mass of the cylinder. (2) The force $-M U \omega \operatorname{Im}(\mathrm{H})$ coswt always opposes the movement of the cylinder and is related to damping mechanism. 
To evaluate the added mass and damping factor, we must compute the value of $H$. From equations (18) and (24), we may write

$$
\begin{aligned}
H= & \left\{2 \alpha^{2}\left[I_{0}(\alpha) K_{0}(\beta)-I_{0}(\beta) K_{0}(\alpha)\right]-4 \alpha\left[I_{1}(\alpha) K_{0}(\beta)+I_{0}(\beta) K_{1}(\alpha)\right]\right. \\
& \left.+4 \alpha \gamma\left[I_{0}(\alpha) K_{1}(\beta)+I_{1}(\beta) K_{0}(\alpha)\right]-8 \gamma\left[I_{1}(\alpha) K_{1}(\beta)-I_{1}(\beta) K_{1}(\alpha)\right]\right\} \\
& \div\left\{\alpha^{2}\left(1-\gamma^{2}\right)\left[I_{0}(\alpha) K_{0}(\beta)-I_{0}(\beta) K_{0}(\alpha)\right]+2 \alpha \gamma\left[I_{0}(\alpha) K_{1}(\beta)\right.\right. \\
& \left.-I_{1}(\beta) K_{0}(\beta)+I_{1}(\beta) K_{0}(\alpha)-I_{0}(\beta) K_{1}(\beta)\right]+2 \alpha \gamma^{2}\left[I_{0}(\beta) K_{1}(\alpha)\right. \\
& \left.\left.-I_{0}(\alpha) K_{1}(\alpha)+I_{1}(\alpha) K_{0}(\beta)-I_{1}(\alpha) K_{0}(\alpha)\right]\right\}-1
\end{aligned}
$$

It is seen that $H$ depends on $\alpha$ and $\beta$ in a very complicated way. While In general, $H$ must be computed from equation (26), simplified results can be obtained in special cases.

1) Infinite, viscous fluid. The value of it for this case can be obtained by taking the limits of Bessel's functions. As $D \sim \infty, \gamma \sim 0$, and $\beta \sim \infty$; then $I_{0}(\beta) \sim \infty, I_{1}(\beta) \sim \infty, K_{0}(\beta) \sim 0$, and $K_{1}(\beta) \sim 0$. With these values, equation (26) reduces to

$$
H=1+\frac{4 \mathrm{~K}_{1}(\alpha)}{\alpha \mathrm{K}_{0}(\alpha)}
$$

2) Infinite, inviscid fluid. If the fluid is inviscld, $\alpha=\infty$ and, from equation (27),

$$
\mathrm{H}=1 \text {. }
$$

This result is consistent with the classical result; the added mass of a cylinder vibrating in an infinite fluid is equal to the mass of the fluid displaced by the cylinder [5].

3) General viscous case with large values of $\alpha$ and $\beta$. For $\alpha$ and $\beta$ very large, the results can be greatly simplified using the asymptotic formulae

$$
\begin{aligned}
& I_{0}(z) \sim \frac{e^{z}}{\sqrt{2 \pi z}}, \\
& I_{1}(z) \sim \frac{e^{z}}{\sqrt{2 \pi z}}, \\
& K_{0}(z) \sim \sqrt{\frac{\pi}{2 z}} e^{-z},
\end{aligned}
$$

and

$$
K_{1}(z) \sim \sqrt{\frac{\pi}{2 z}} e^{-z}
$$


As a result of these approximations, $H$ becomes

$$
H=\frac{\left[\alpha^{2}\left(1+\gamma^{2}\right)-8 \gamma\right] \sinh (\beta-\alpha)+2 \alpha\left(2-\gamma+\gamma^{2}\right) \cosh (\beta-\alpha)-2 \gamma^{2} \sqrt{\alpha \beta}-2 \alpha \sqrt{\frac{\alpha}{\beta}}}{\alpha^{2}\left(1-\gamma^{2}\right) \sinh (\beta-\alpha)-2 \alpha \gamma(1+\gamma) \cosh (\beta-\alpha)+2 \gamma^{2} \sqrt{\alpha \beta}+2 \alpha \sqrt{\frac{\alpha}{\beta}}} .
$$

4) General inviscid case with large values of $\alpha$ and $\beta$. If the fluid is inviscid, equation (30) reduces to

$$
H=\frac{1+\gamma^{2}}{1-\gamma^{2}} \text {. }
$$

Equations (27), (28), (30) and (31) are useful for the special cases cited. For the general case, values of $\mathrm{H}$ have been computed from equation (26) in terms of two parameters, $s\left(=\frac{\omega d^{2}}{v}\right)$ and radius ratio $D / d$. From equation (17),

$$
\alpha=\sqrt{1 \mathrm{~s}} \text {, }
$$

and

$$
\beta=\frac{D}{d} \sqrt{i s}
$$

The real and imaginary parts of $H$ are plotted in Figs. 2 and 3, respectively, as functions of the radius ratio $(D / d)$ for selected values of the nondimensional parameter S.

\section{Added Mass and Damping Factors}

The added mass correction factor $\mathrm{C}_{\mathrm{N}}$ is defined as a constant which multiplies the mass of displaced fluid to give the added mass. From equation (25), by definition,

$$
C_{M}=\operatorname{Re}(\mathrm{H}) \text {. }
$$

Similarly, from equation (25), if MUwIm(H) is considered as the amplitude of a viscous damping force, by definition the damping coefficient can be written as

$$
C_{v}=\operatorname{Min}(H)
$$

The equivalent viscous damping factor $\zeta_{\mathrm{n}}$ is defined as the ratio of the viscous damping coefficient to the critical damping coefficient

$$
\zeta_{\mathbf{n}} \equiv \mathrm{C}_{\mathbf{v}} / \mathrm{C}_{\mathrm{CR}}
$$

where, $C_{C R}=2\left(C_{M} M+m\right) \omega_{n} ; \omega_{n}$ is rod natural frequency. When oscillating at the rod natural frequency, $\omega=\omega_{n}$ and

$$
\zeta_{n}=\frac{1}{2}\left(\frac{M}{C_{M} M+m}\right) I m(H)
$$




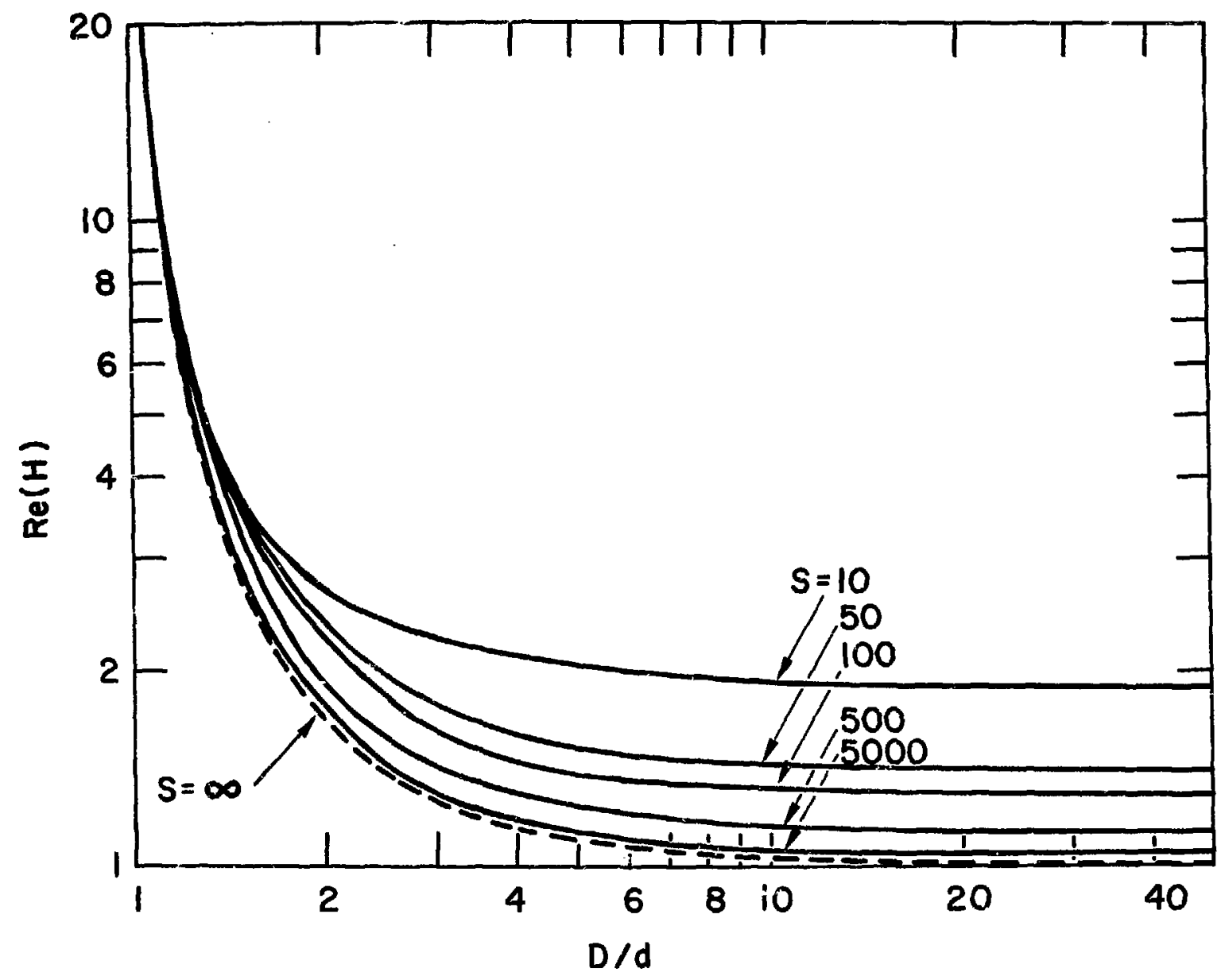

Fig. 2. Real Values of $H$ as a Function of $D / d$ for Selected Values of $S$ 


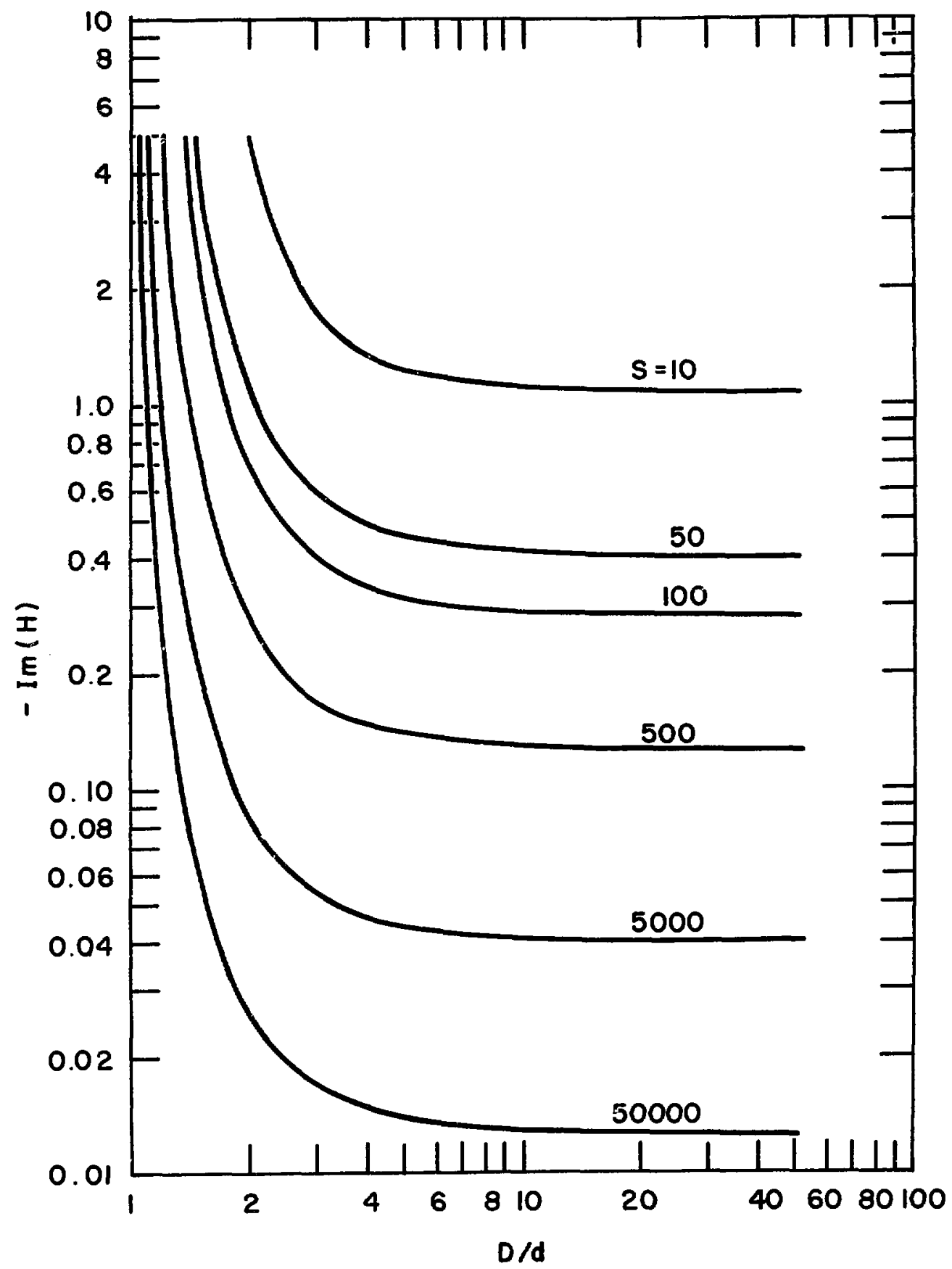

Fig. 3. Imaginary Values of $\mathrm{H}$ as a Function of $\mathrm{D} / \mathrm{d}$ for Selected Values of $S$ 


\section{EXPERIMENT}

In Section II of the report, theoretical results are derived giving the added mass correction factor and equivalent viscous damping factor (equations (33) and (36), respectively) for a flexible rod vibrating in a fluid annulus. Situations which also occur involve the vibration of rods or tubes grouped in bundles, for example, reactor fuel pins or heat exchanger tubes. The question arises as to what effect the adjacent components have on added mass and damping. To evaluate the theoretical results derived in Section II, and to obtain insight into the effect of adjacent elements, a series of experiments with cantilevered rods as the vibrating elements was designed and performed.

\section{A. Description of Experiment}

The test element consists of a 0.5 inch (12.7mm) diameter aluminum rod fixed to a base plate to achieve cantilever end conditions. An annular region around the rod is formed by a 2.5 inch $(63.5 \mathrm{~mm}) \mathrm{i}$. d. circular cylindrical containment: shell mounted to the base plate, concentric with the rod. Two series of tests were performed. In the one series, the width of the annulus was varied by inserting machined brass liners into the containment she11; outer diameters of the annular gaps so obtained are as follows: 2.5 (63.5), $2.25(57.2), 2.0(50.8), 1.75(44.4), 1.5(38.1), 1.25$ (31.8), $1.0(25.4), 0.75(19.0)$, and $0.625(15.9)$ inches (mm). In the second series of tests the effect of "rigid" adjacent rods of the same diameter, mounted in a hexagonal pattern was investigated; the pitch-to-diameter ratios tested are as follows: $1.75,1.50,1.25$, and 1.12. A complete set of tests was performed with a 28.1 inch $(0.714 \mathrm{~mm})$ long test element; the rod was then cut to a 14 inch $(0.356 \mathrm{~m})$ length and tests in water were performed to assess th. affect of frequency on added mass and damping.

A means to excite the rod is provided by an electromagnetic exciter assembly. This exciter assembly consists of 13 permanent magnets, $1 / 4$ inch $(6.35 \mathrm{~mm})$ in diameter by $3 / 8$ inches $(9.52 \mathrm{~mm})$ long, embedded in the aluminum rod approximately 2.7 inches $(68.6 \mathrm{~mm})$ from the free end, and a pair of $350-$ turn coils of no. 26 wire attached to the outside of the containment shell, conforming to it: curvature. An alternating current applied to the coils from a signal generator induces an alternating magnetic field which, in turn, produces a force on the rod through the embedded magnets.

Response, in the form of rod displacement, is measured using an electro-optical displacement tracker mounted above the test assembly and adjusted to track a black/white interface painted on the end of the rod. RMS current to the coil which is proportional to input force, is measured on an ammeter. A servo system can be used to control either displacement output, or current input, as desired. The data is processed on a fast Fourier transform analyzer.

Tests were performed with four different fluids: air, water, mineral oil, and silicone oil. Fluid properties are listed in Table 1. 0il viscosities were measured at room temperature using a capillary-type viscometer. 
TABLE I TEST FLUID PROPERTIES

\begin{tabular}{lcc}
\hline Test Fluid & V1scosity, $\mu(\mathrm{cP})$ & Specifle Gravity, \\
\cline { 2 - 3 } AIR & 0.018 & 0.00121 \\
HATER & 1.0 & 1.0 \\
MINERAL OIL & 41 & 0.935 \\
SILICONE OIL & 145 & 0.956 \\
\hline
\end{tabular}

Approximately 10 measurements were taken at varlous times during a given test and the results averaged.

B. Measurement Methods

The quantities of interest are the added mass correction factor and equivalent viscous damping factor assoclated with that portion of the fluld reaction force which acts to oppose velocity of the rod.

1) Added Mass Correction Factor

The natural frequency of a cantllever rod vibrating in a fluld can be written in the form

$$
f_{n}=\frac{1}{2 \pi} \frac{a^{2}}{\ell^{2}} \sqrt{\frac{E I}{m+G_{M}}}
$$

where, a is a mode constant, $\ell$ is length, EI is flexural rigidity, and $m$ is rod mass per unit length. Vibrating in air, $m>C_{M}$, and equation (37) becomes

$$
\left(f_{n}\right)_{A I R^{-}}=\frac{1}{2 \pi} \frac{a^{2}}{l^{2}} \sqrt{\frac{E I}{m}} .
$$

Solving equation (37) for $C_{M}$ and using equation (38) obtains

$$
C_{M}=\frac{m}{M}\left\{\left[\frac{\left(f_{n} \mid A I R\right.}{f_{T}}\right]-1\right\} \text {. }
$$


Knosing the mass per unit length of the rod (m) and displaced mass of fluid per unti: length $(M)$, equation (39) can be used to compute $G$ from measured values of resonant frequencies. This approach assumes that any changes in resonant frequency caused by changes in damping are negligible in comparison with the change caused by added mass.

The rod does not have uniform mass per unit length because of the permanent magnets embedded near the free end. Consequently, in equations (37)(39), m must be considered as an effectlve mass per unit length which accounts for the mass of the magnets. The effectlve mass per unit length is evaluated in Appendix $A$ for the two test element lengths. Expressing the mass of displaced fluid in terms of specific gravity, $\Gamma$, and defining

$$
\mathbf{R}=\mathrm{m} / \mathrm{M}_{\text {water }}
$$

yields

$$
G_{M}=\frac{R}{\Gamma}\left\{\left[\frac{\left(f_{n}\right)_{A I R}}{\left(f_{n}\right)}\right]^{2}-1\right.
$$

where values for $\Gamma$ and $R$ are given in Tables 1 and 2 respectively.

\section{Equivalent Viscous Damping Factor}

Energy loss mechantsms for a rod vibrating in a fluld include materlal damping, assoclated with internal friction in the solid material, fluld drag forces due to the viscosity of the fluid, and losses at the support location. In general, for the test element under consideration, material damping and damping assolcated with losses at the support are small compared with fluld viscosity effects in liquids. Damping can be measured and calculated by several different methods including $\log$ decrement from autocorrelation of response to white noise input or from a "pluck" test, magnification factor (Q) at resonance, bandwidth of frequency response function, and measurement of input power at resonance. The bandwidth method was the primary method used in these experiments. The advantages of the bandwidth method over the $10 \mathrm{~g}$ decrement method and pluck test is that higher modes are not Involved and response levels can be readily controlled, if amplitude dependency is suspected. The advantage over the magnification factor method is that only the shape of the curve is involved, calibration factors and the fact that energy may go into different modes is not of concern. Damping is readily obtained from the transfer function, or frequency response curve, as

$$
\zeta=\frac{1}{2 \sqrt{N^{2}-1}} \frac{\Delta f_{N}}{f_{n}},
$$

where

$$
\Delta f_{N}=f_{N}^{(1)}-f_{N}^{(2)}
$$


$f_{n}$ is the natural (resonant) frequency, and $f_{N}^{(1)}$ and $f_{N}^{(2)}$ are the frequencles at which the response is a factor $(1 / N)$ th of the resonant response. Since $\Delta f_{N}$ is generally the difference between two closely spaced numbers, the greater the value of $\Delta f_{\mathbb{N}}$, the greater the accuracy of the measurement.

\section{Test Procedures and Results}

Virtual mass is computed from measured values of resonant frequencies. Damping factor is computed from the frequency response function of the rod. For each of the two test element lengths, rod natural frequency and damping in air is measured. In-air results are giveis in Table 2. It should be noted that the in-air natural frequency of the 28.1 inch $(0.714 \mathrm{~m})$ test element was only measured once, prior to Test No. 1. During tests with the 14 inch $(0.356 \mathrm{~m})$ test element, five measurements of rod natural frequency in-air were made with an averaged deviation from the mean of 0.24 per cent.

For a given annular gap and fluld, resonant frequency is determined by applying a sinusoidal current to the colls, slowly sweeping through the frequency range centered about the fundamental frequency of the rod, selecting the peak response, recording the perlod associated with the peak oscillation as read from a frequency meter, and taking the reciprocal of the perfod to obtain resonant frequency. Four independent mearurements are obtained by traversing two cycles of increasing and decreasing annular gaps and at each gap approaching resonant frequency by sweeping both up and down in frequency. The four measurements are compared to assess repeatability and, if repeatability is shown, are then averaged. The averaged experimentally-determined resonant frequencies are summarized in Table 2. Added mass correction factor is computed from equation (41) using the results given in Table 2, and is also tabulated in Table 2 along with Stokes number (S) based on the resonant frequency of the rod and rod radius.

Damping factor is obtained by applying random excitation to the rod, computing the transfer function, and calculating damping factor from the transfer function by both the bandwidth and magnification factor methods. The transfer function is computed from the cross-spectral density function and its validity is checked by computing the coherence function; both computations are performed on a digital fast Fourier transform analyzer. At each test condition, damping factor is computed from the baniwidth method at two different response levels corresponding to $\mathrm{N}$ equal to $\sqrt{2}$ and 2 in equation (42). Damping factor is also computed from the magnification factor at resonance $(Q)$, corrected to account for calibration uncertainties and energy which goes into sustaining vibration in the plane normal. to the plane of excitation. The three values of damping factor are then compared to assess relfability of the measurements and, if agreement is good, they are averaged. It is assumed that the in-air measurements of damping account primarily for material and joint damping. Consequently, to obtain a more accurate measure of the viscous damping, the measured value of in-air damping is subtracted from the experimentally determined values accounting for the differences in critical damping coeffictent. Resuits are sumarized in Table 2. 
TABLE 2 SUMMARY OF TEST RESULTS

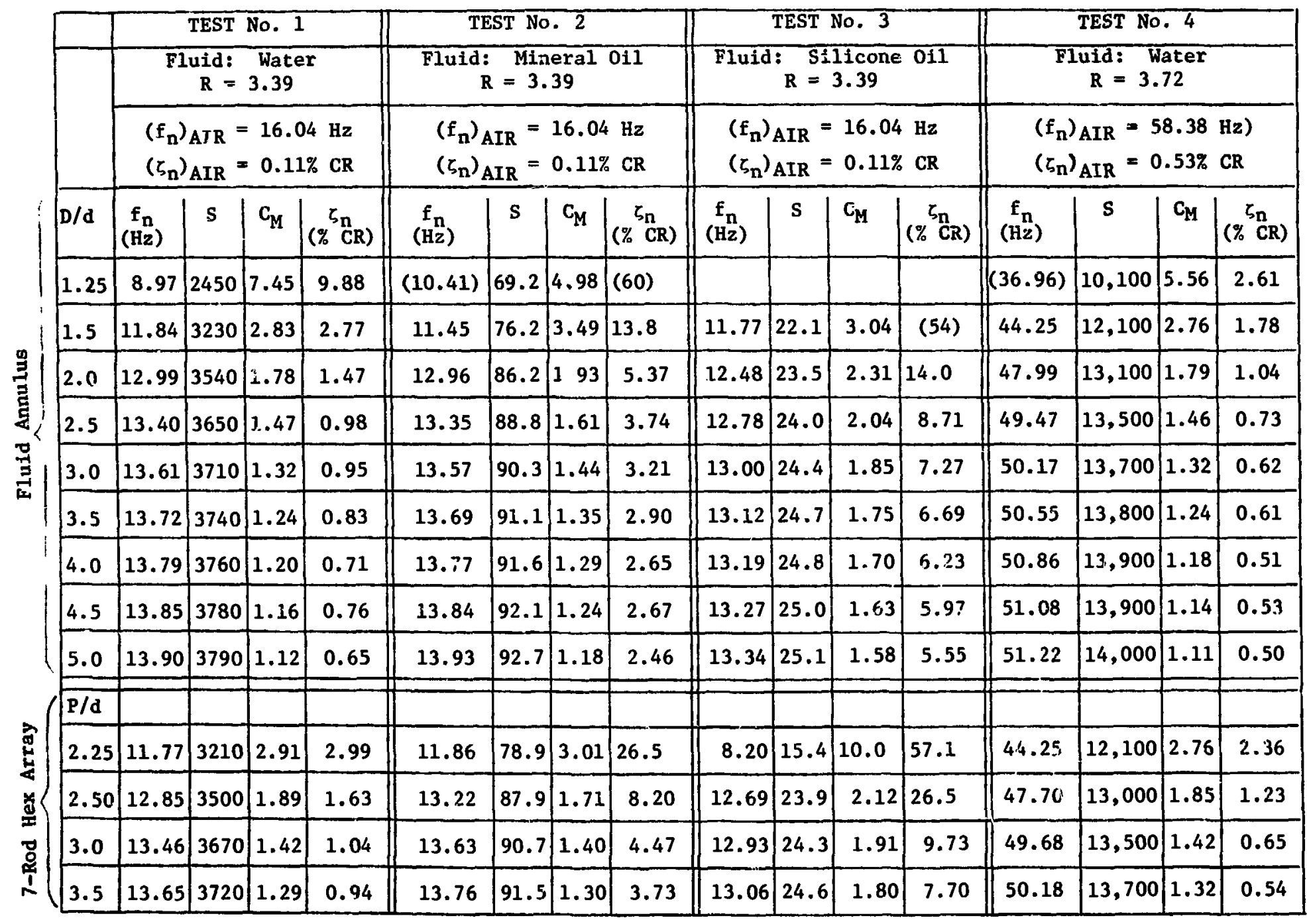


Theoretical values of $\operatorname{Re}(H)\left(=C_{H}\right)$ and $\operatorname{Im}(H)$ are obtained using the Stokes Numbers and radius ratlos given in Table 2. "Theoretical" values of damping factor are computed from equation (36), rewritten in the form

$$
\zeta_{n}=\frac{1}{2}\left(\frac{1}{C_{M}+R / \Gamma}\right) I m(H),
$$

using theoretical values of $C_{M}$ and values of $\Gamma$ and $R$ taken from Tables 1 and 2. In Figs. 4 and 5, theoretical and experimental results are compared.

Agreement between theoretical and experimental results are very good for the tests with water (Tests No. 1 and 4); among other things, the results Indicate the decrease in added mass and damping with increasing frequency as predicted by theory. In Fig. 4, while the experimental results from Tests No. 2 and 3 exhibit the proper trends, the agreement with theory is not as good. However, as noted above, only one measurement of the "in-air" natural frequency of the 28.2 inch $(0.714 \mathrm{~m})$ test element was made at the initiation of testing. Since calculation of the added mass correction factor is sensitive to the valus of in-air natural frequency, the experimental phase of the study is deficient in that additional measurements of in-air natural frequencles were not made. In an attempt to compensate for this deficiency, it is observed that it is reasonable to expect slight changes in frequecny (on the order of 1 or 2 per cent) caused by "disturbing" the test assembly in changing test fluids. Based on the good agreement between theory and experiment for the water tests (for which in-air measurements were made prior to testing), assume that the theory is valid for a rod vibrating in an "Infinite" fluid and that a radius ratio $(D / d)$ of 5 approximates the infinite condition. With the values of $\Gamma$ and $R$ gtven in Table 1 and 2 , respectiveiy, it is easy to use equation (41) to calculate the following values of in-air natural frequencies that result in agreement between theoretical and experimental results at $\mathrm{D} / \mathrm{d}=5$ for Tests No. 2 and 3 :

$$
\left(f_{n}\right)_{\text {AIR }}= \begin{cases}16.43 \mathrm{~Hz}, & \text { Test } 2 \text { (Mineral 011) } \\ 16.26 \mathrm{~Hz}, & \text { Test } 3 \text { (Sillcone 0i1) }\end{cases}
$$

In Fig. 4, "experimental" results obtained from equation (41), using measured values of resonant frequency from Tests 2 and $3 \mathrm{given}$ in Table 2, and the appropriate values of in-air natural frequencles as given by equation (44), are compared with theory. It is readily observed that agreement is now very good over the range of radius ratios and Stokes numbers tested. This leads one to suspect that some of the discrepancy between theory and experiment in Tests No. 2 and 3 might be attributed to slight changes in in-air natural frequency caused by disturbing the base piate between tests.

Results from the 7-rod hexagonal array tests are also sumarized in Table 2 for the four different pitch-to-diameter ratios tested. Tests were performed with the center rod excited in-line with adjacent rods and also 


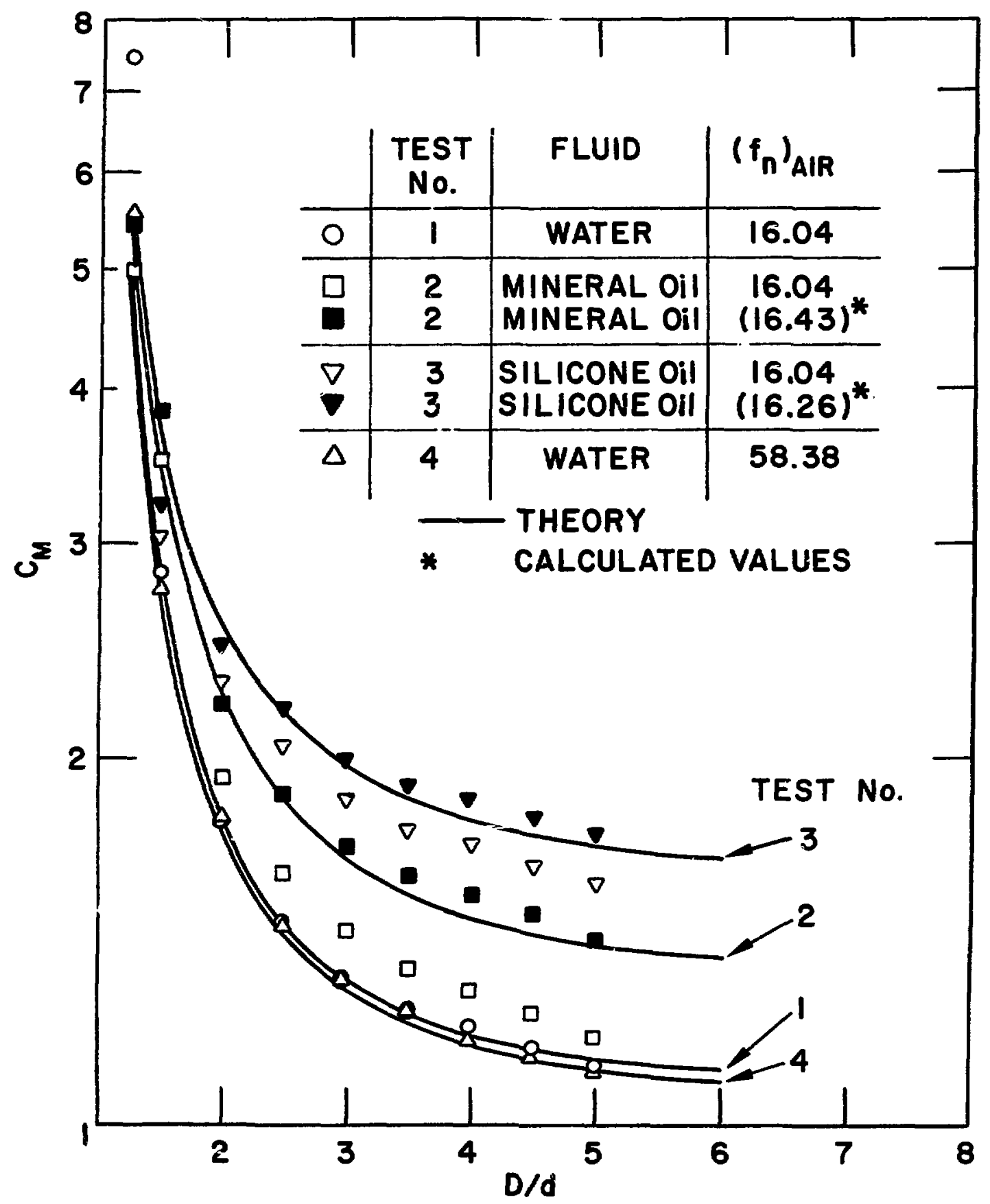

F1g. 4. Theoretical and Experimental Values of $C_{M}$ as a Function of $D / d$ 


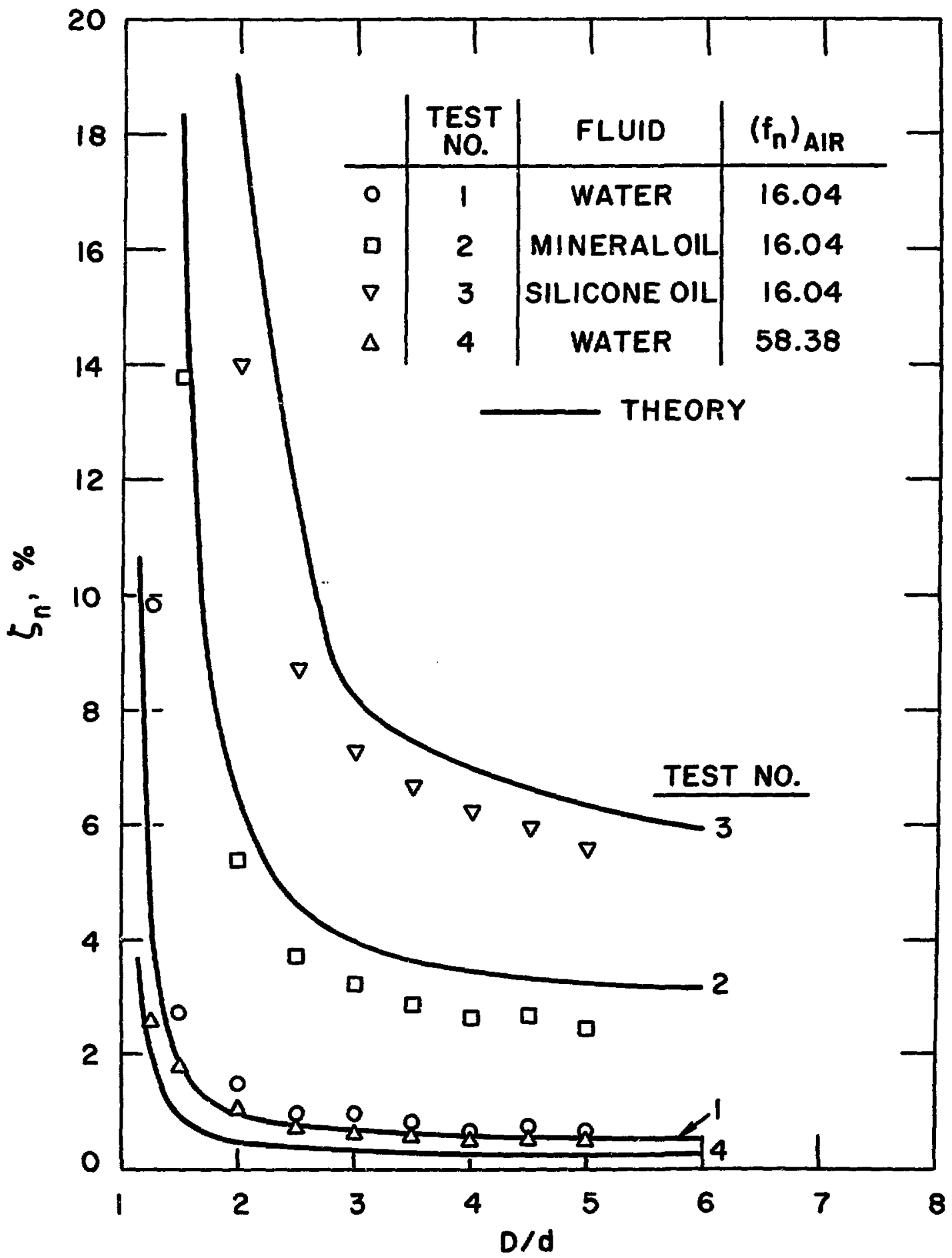

F1g. 5. Theoretical and Experimental Values of $\zeta_{n}$ as a Function of $\mathrm{D} / \mathrm{d}$ 
in a plane $30^{\circ}$ to the in-1ine direction. Results from the two series of tests were essentially the same; the presented results represent averaged values. In an attempt to correlate che 7-rod hexagonal array results with those from the fluid annulus tests an effective radius ratio was determined from theory and a correction factor to the pitch-to-radius ratio was defined as

$$
C_{D}=\frac{(D / d) \text { effective }}{(\mathrm{P} / \mathrm{d})}
$$

The results are given in Fig. 6 as curves of $C_{D}$ versus $P / d$. Unfortunately, a constant value of $C_{D}$ is not obtained and, further, the correction factor is different for the added mass and damping factor. Nevertheless, the results give insight into the behavior of closely-spaced rod arrays, thus providing some design guidance prior to the development of a theory.

\section{CONCLUDIN/ REMARKS}

In this report, a closed form solution for the added mass and damping coefficient is obtained and a series of experiments is conducted for a cylindrical rod vibrating in a viscous fluid filling a fixed cylindrical shell. Analytical results and experimental data for the added mass coefficient and damping ratio are found to be in good agreement. Thus, the closed form solution can be used in the study of the response of circular cylindrical rods in viscous fluid annuli.

A few remarks should be made:

(1) The added mass factor $C_{M}$ and damping coefficient $C_{v}$ are dependent on the dimensionless number $S\left(=\frac{\omega d^{2}}{v}\right)$; as $S$ increases, $C_{M}$ and $C_{v}$ decrease. For fixed values of $d$ and $v, c_{M}$ decreases with an increasing $w$; this behavior is also noted by Miller [7]

(2) In the anaiysis, the fluid field is considerad to be two dimensional; that is, the axial motion of the fluid is neglected. This is justified for a large value of $\ell / d$ as in the case of inviscid fluid [12]. When $\ell$ and $d$ are of the same order of magnitude, the three dimensional effect of the flow should be considered.

(3) The linear theory presented in this report is based on the assumption that the vibration amplitude is small. As the rod displacement becomes large, the nonilinear effects of the fluid fileld w11 be important. In fact, it was shown by Miller [7] that $C_{M}$ is a function of the vibration amplitude. However, for small vibration, $\mathrm{M}$ in the cases of many reactor components, the linear theory is applicable, and $C_{M}$ and $C_{V}$ are independent of the amplitude of oscillation. 


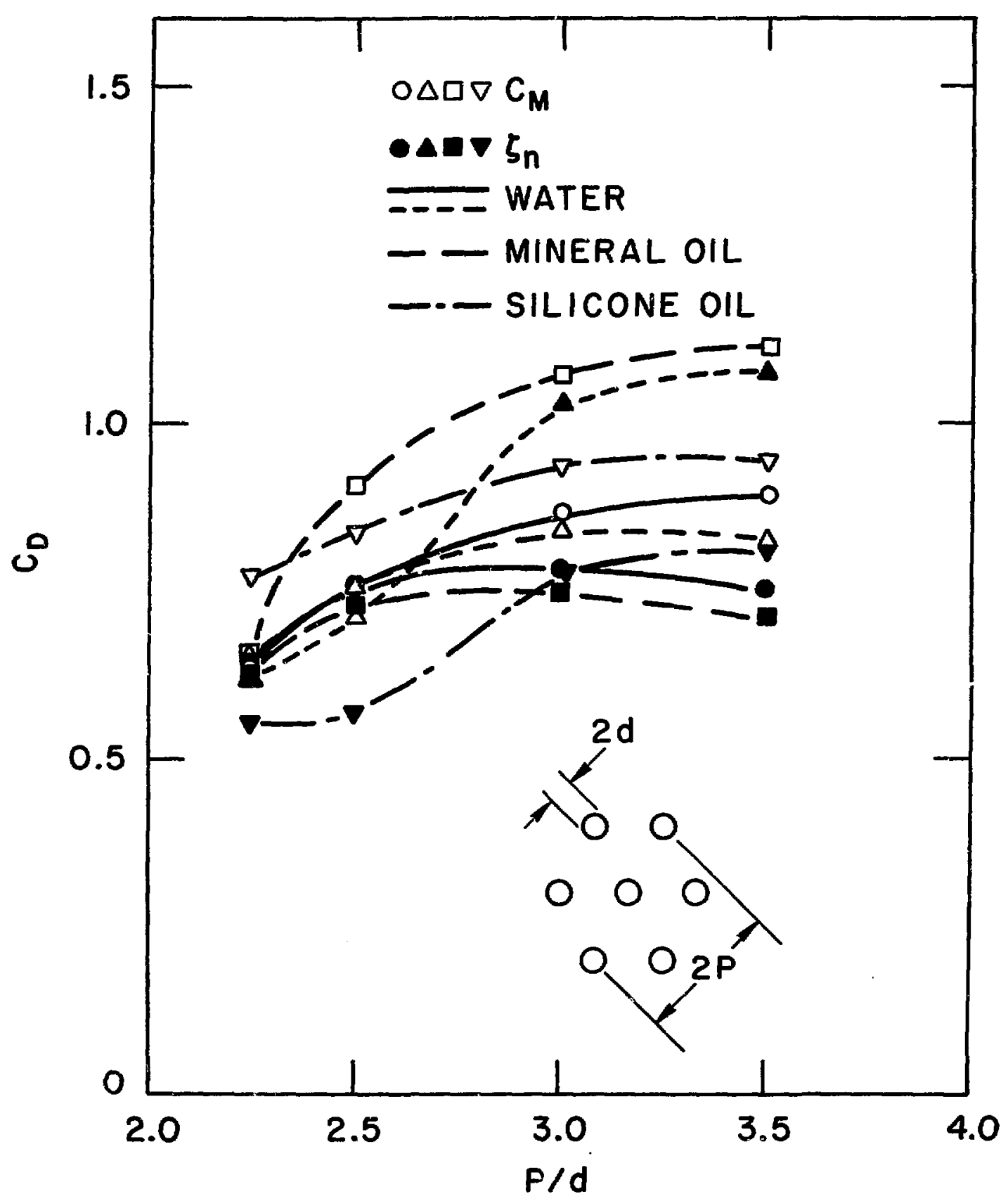

Fig. 6. Effective-Radius Ratio for Hexagonal Array of Rods 


\section{APPENDIX}

\section{Effective Mass Per unit Length of Rod}

The permanent magnets embedded near the free end of the cantilever represent a concent rated mass that must be accounted for in computing the effective mass per unit length of the test element. Employing the RayleighRitz method, the natural frequency of a uniform beam of length $\ell$, with a concentrated mass $\left(M_{0}\right)$ located at $x=b$ can be written in the form

$$
\omega_{n}^{2}=\frac{E I \int_{0}^{l}\left[\phi^{\prime \prime}(x)\right]^{2} d x}{\rho A \int_{0}^{l} \phi^{2}(x) d x+M_{0} \phi^{2}(b)},
$$

where $\phi(x)$ is the mode shape. Nondimensionalizing $x$ as $\bar{x}=x / \ell$ and defining

$$
a^{4}=\frac{\int_{0}^{1}\left[\phi^{\prime \prime}(\bar{x})\right]^{2} d \bar{x}}{\int_{0}^{1} \phi^{2}(\bar{x}) d \bar{x}}
$$

and

$$
k=\frac{\phi^{2}(\bar{b})}{\int_{0}^{1} \phi^{2}(\bar{x}) d \bar{x}},
$$

allows writing equation (46) in the form

$$
f_{n}=\frac{1}{2 \pi} \frac{a^{2}}{l^{2}} \sqrt{\frac{E I}{m}}
$$

where

$$
m=\rho A\left(1+\frac{M_{o}}{\rho A l} k\right) \text {. }
$$

The effective mass of the magnets, $M_{0}$, is given by

$$
M_{0}=\left(\rho_{\text {steel }}-\rho_{a 1} v_{0}\right.
$$

where $v_{0}$ is the volume occupied by the magnets. 
For a 0.5 inch $(12.7 \mathrm{~mm})$ diameter aluminum rod

$$
\rho A=0.498 \times 10^{-4} 1 \mathrm{~b} \cdot \mathrm{sec}^{2} / \mathrm{m}^{2}(0.171 \mathrm{~kg} / \mathrm{m}) \text {. }
$$

There are 130.25 inch (6.35 $\mathrm{mm})$ diameter, 0.375 inch $(9.52 \mathrm{~mm})$ long magnets with an effective mass as obtained from equation (51), given by

$$
M_{0}=1.19 \times 10^{-4} 1 \mathrm{~b} \cdot \sec ^{2} / \mathrm{m}(0.821 \mathrm{~kg} / \mathrm{m})
$$

With the center of the magnet assembly located 2.7 inch $(68.6 \mathrm{~mm})$ from the free end of both the 28.1 inch $(0.714 \mathrm{~m})$ and 14 Inch $(0.356 \mathrm{~m})$ Iong test elements, the corresponding values of $k$, assuming the mode shape to be that of a uniform cantilever beam, are 2.96 and 2.19 respectively. Evaluating the effective mass by using the appropriate $k$ values, and substituting equations (50) and (51) into equation (48), yields

$$
\text { If }=\left\{\begin{array}{l}
0.622 \times 10^{-4} 1 \mathrm{~b} . \sec ^{2} / 1 \mathrm{n}^{2}(0.429 \mathrm{~kg} / \mathrm{m}), \ell=28.1 \text { inch }(0.714 \mathrm{~m}) \\
0.682 \times 10^{-4} \mathrm{Ib} . \sec ^{2} / 1 \mathrm{n}^{2}(0.470 \mathrm{~kg} / \mathrm{m}), \ell=14 \text { inch }(0.356 \mathrm{~m})
\end{array}\right.
$$




\section{REFERENCES}

1. Chen, S. S. and Wambsganss, M. W., Parallel-Flow Induced Vibration of Fuel Rods, Nucl. Eng. and Des. 18, 1972, pp. 253-278.

2. Connors, H. J., Fluidelastic Vibration of Tube Arrays Excited by Cross Flow, Symposium of Flow-Induced Vibrations in Heat Exchangers, ASME Winter Annual Meeting, Dec. 1970, pp. 42-56.

3. Stokes, G. G., On Some Cases of Fluid Motion, Proceedings Cambridge Philosophical Society, Vol. 8, May 1943, pp. 105-137.

4. Muga, B. J., and Wilson, J. F., Dynamic Analysis of Ocean Structures, Plenum Press, New York-I.ondon (1970).

5. Patton, K. T., Tables of Hydrodynamic Mass Factors for Translational Motion, ASME Paper No. 65-WA/UNT-2.

6. Ackermann, N. T., and Arbhabhirama, A., Viscous and Boindary Effects on Virtual Mass, J. Eng. Mech. Div., ASCE, Vol. 90, EM4, 1964, pp. 123-130.

7. Miller, R. R., The Effects of Frequency and Amplitude of Oscillation on the Hydrodynamic Masses of Irregular Shapes Bodies, M. S. Thesis, Untv. of Rhode Island, 1965.

8. Fritz, R. J., and Kiss, E., The Vibration Response of a Cantilevered Cylinder Surrounded by an Annular Fluid, KAPL-M-6539, Feb. 24, 1966; available from Clearinghouse for Federal Scientiflc and Technical Information, U. S. Department of Commerce, Springfield, Va.

9. Fritz, R. J., The Effects of an Annular Fluid on the Vibrations of a Long Rotor, Part 1 - Theory; and Part 2 - Test, J. Basic Engineering, Trans. ASME, Series D, Vol. 92, No. 4, Dec. 1970, pp. 923-937.

10. Keane, J. A., On the Elastic Vibration of a Circular Cantilever Tube in a Newtonian Fluid, Ph.D. Thesis, Carnegie Institute of Technology, Sept. 1963.

11. Schlichting, H., Boundary Layer Theory, McGraw-Hill Book Company, N. Y., 1960.

12. Chen, S. S., Dynamics of a Rod-Shell System Conveying PZuid, to appear In Nuc1. Eng. Design. 